\title{
THE TAYLOR SERIES EXPANSION COEFFICIENTS FOR SOLUTIONS OF THE EMDEN-FOWLER TYPE EQUATIONS
}

A. GRITSANS ${ }^{1}$ and F. SADYRBAEV ${ }^{2}$

${ }^{1}$ Daugavpils University

Daugavpils, Parades str. 1

E-mail: arminge@dau.lv

${ }^{2}$ Institute of Mathematics and Computer Science, University of Latvia

Riga, Rainis blvd 29

E-mail: felix@cclu.lv

Received September 24, 2004; revised June 12, 2005

Abstract. We present the explicit non-recursive formulas for the Taylor series expansion coefficients for the functions $S_{n}(t)$ defined as solutions of the Emden Fowler type equations $x^{\prime \prime}=-n x^{2 n-1}$ with the initial conditions $x(0)=0, x^{\prime}(0)=1$, where $n=1,2, \ldots$

Key words: Emden - Fowler equations, Taylor series expansion, lemniscatic sine

\section{Introduction}

In this note we give the expressions of the Taylor series expansion coefficients for the lemniscatic sine function and similar ones. The lemniscatic function sl $t$ can be defined as

$$
t=\int_{0}^{y} \frac{\mathrm{d} s}{\sqrt{1-s^{4}}}, \quad y=\operatorname{sl} t
$$

on the interval $[0, A]$, where $A=\int_{0}^{1} \frac{\mathrm{d} s}{\sqrt{1-s^{4}}}$, and then extended to entire $\mathbb{R}$ in a sin-like manner. The integral in (1.1) appears in the theory of the lemniscate curve given by $\left(x^{2}+y^{2}\right)^{2}=x^{2}-y^{2}$. The alternative way to define the function $\mathrm{sl} t$ is to consider the nonlinear Cauchy problem

$$
x^{\prime \prime}=-2 x^{3}, \quad x(0)=0, x^{\prime}(0)=1 .
$$


It is known ([9][§22.8]) that the lemniscatic sine function sl $t$ can be expressed in terms of the Jacobian elliptic functions ([1][Ch. 16]) as

$$
\operatorname{sl} t=k_{0} \operatorname{sd}\left(\frac{t}{k_{0}} ; k_{0}\right)=k_{0} \frac{\operatorname{sn}\left(\frac{t}{k_{0}} ; k_{0}\right)}{\operatorname{dn}\left(\frac{t}{k_{0}} ; k_{0}\right)}, \quad k_{0}:=2^{-\frac{1}{2}}
$$

or

$$
\operatorname{sl} t=\operatorname{sn}(t ; i), \quad i=\sqrt{-1} .
$$

As far as we know there are no formulas for the general term of the Taylor series expansion for the Jacobian elliptic functions. In seventieth of the XXth century Schett in [6], [8] and [7] provided the recurrent formulas for the Taylor series expansion coefficients of the Jacobian elliptic functions. Dumont in [2] has interpreted these results from combinatorial point of view. Myung in [4] considered Schett's functions $X, Y, Z$ and provided some recursive formulas using the algebraical approach. Wrigge in [10] considered the integral definition of $\operatorname{sn}(t ; k)$, namely

$$
t=\int_{0}^{\operatorname{sn}(t ; k)} \frac{\mathrm{d} s}{\sqrt{\left(1-s^{2}\right)\left(1-k^{2} s^{2}\right)}} .
$$

Expanding the integrand and then integrating we get that

$$
t=\sum_{n=0}^{\infty} C_{2 n}(k) \frac{\operatorname{sn}^{2 n+1}(t ; k)}{2 n+1}, \quad \text { where } \quad C_{2 n}(k)=k^{n} P_{n}\left(\frac{k}{2}+\frac{1}{2 k}\right)
$$

and $P_{n}(s)$ is the $n$-th Legendre polynomial. Using Bürmann-Lagrange's inversion formula [1], one obtains the Taylor series expansion

$$
\operatorname{sn}(t ; k)=\sum_{n=0}^{\infty} \frac{t^{2 n+1}}{(2 n+1) !} \sigma_{2 n+1}(k),
$$

where

$$
\sigma_{2 n+1}(k)=\left[\frac{\mathrm{d}^{2 n}}{\mathrm{~d} s^{2 n}}\left(\frac{1}{P(s)}\right)^{2 n+1}\right]_{s=0}, \quad P(s)=\sum_{m=0}^{\infty} C_{2 m}(k) \frac{s^{2 m}}{2 m+1} .
$$

The author discusses then the computational complexity of this method and provides the "more practical" recurrence formulas for the Taylor series coefficients of $\mathrm{sn}(t ; k)$ and $\mathrm{sn}^{2}(t ; k)$. The first nine nontrivial coefficients were computed. In the work [11] the expansions of $\operatorname{sn}(t ; k)$ and $\operatorname{sn}^{2}(t ; k)$ were considered in powers of the modulus $k$. Closed expressions for the first nontrivial coefficients were obtained.

In this paper we obtain the formulas for the Taylor series coefficients for the function sl $t$ and similar functions $S_{n}(t)$ using the same approach. The functions are defined as solutions of the initial value problems 


$$
x^{\prime \prime}=-n x^{2 n-1}, \quad x(0)=0, \quad x^{\prime}(0)=1,
$$

where $n=1,2, \ldots$ First we consider the function $t=t(y)$ defined by (1.1) or similar integral relations. Then we expand this function at zero point using the binomial formula. The coefficients for the Taylor series for sl $t$ (and $S_{n}(t)$ ) are obtained by inversion of those in the Taylor series expansion for $t=t(y)$. For this, we use the recent formula by Zabreiko - Lysenko [12].

\section{Main result}

Theorem 1. The nontrivial coefficients of the Taylor series expansion for the function $S_{n}(t)=\sum_{m=0}^{\infty} S_{n, m} t^{m}$ are given by $S_{n, 1}=1$ and

$$
\begin{aligned}
S_{n, 2 n k+1}=\frac{1}{2 n k+1} \sum_{1 \beta_{1}+\cdots+k \beta_{k}=k} & (-2)^{-2 k+\sum_{j=1}^{k} \beta_{j}} \\
& \times\left(V ; \beta_{1}, \ldots, \beta_{k}, 2 n k\right) \frac{\prod_{j=1}^{k}\left(\begin{array}{c}
2 j-1 \\
j-1
\end{array}\right)}{\prod_{j=1}^{k}(2 n j+1)^{\beta_{j}}},
\end{aligned}
$$

where $k=1,2, \ldots$ and

$$
\left(V ; \beta_{1}, \ldots, \beta_{k}, 2 n k\right)=\frac{\left(\beta_{1}+\beta_{2}+\cdots+\beta_{k}+2 n k\right) !}{\beta_{1} ! \beta_{2} ! \cdots \beta_{k} !(2 n k) !}, \quad\left(\begin{array}{c}
2 j-1 \\
j-1
\end{array}\right)=\frac{(2 j-1) !}{j !(j-1) !} .
$$

Proof. Let $F_{n}(x)$ be the inverse function of $S_{n}(t)$. Thus

$$
F_{n}(x)=\int_{0}^{x} \frac{d u}{\sqrt{1-u^{2 n}}}=\sum_{k=0}^{\infty} F_{n, 2 n k+1} x^{2 n k+1}
$$

in some neighbourhood of zero, where

$$
\begin{aligned}
& F_{n, 2 n k+1}=\frac{F_{n}^{(2 n k+1)}(0)}{(2 n k+1) !}=\frac{(2 k-1) ! !}{(2 k) ! !} \cdot \frac{1}{2 n k+1} \quad(k=0,1,2, \ldots) \\
& (2 k-1) ! !=1 \cdot 3 \cdot 5 \cdots(2 k-1) \quad(k=0,1,2, \ldots), \quad(-1) ! !=1 \\
& (2 k) ! !=2 \cdot 4 \cdot 6 \cdots 2 n \quad(k=0,1,2, \ldots), \quad 0 ! !=1 .
\end{aligned}
$$

Let us consider $y=f(x)$ and let $x=g(y)$ be an inverse function. It was shown in the recent article of Zabreiko and Lysenko [12] that the Ostrowski's formula [5] for the $m$-th derivative of an inverse function can be written as

$$
\begin{aligned}
& g^{(m)}(y)= \sum_{1 \alpha_{2}+\cdots+(m-1) \alpha_{m}=m-1}(-1)^{\alpha_{2}+\cdots+\alpha_{m}} \frac{\left(2 \alpha_{2}+\cdots+m \alpha_{m}\right) !}{\alpha_{2} !(2 !)^{\alpha_{2}} \cdots \alpha_{m} !(m !)^{\alpha_{m}}} \\
& \times\left[\left(f^{\prime}(x)\right)^{-1}\right]^{2 \alpha_{2}+\cdots+m \alpha_{m}+1}\left(f^{(2)}(x)\right)^{\alpha_{2}} \cdots\left(f^{(m)}(x)\right)^{\alpha_{m}}
\end{aligned}
$$


where $y=f(x), f^{\prime}(x) \neq 0$. We will refer to this formula as the OZL-formula.

Let $g=S_{n}$ and $f=F_{n}$. Notice that $F_{n}{ }^{\prime}(0)=1$. It appears that

$$
S_{n}(t)=\sum_{m=0}^{\infty} S_{n, m} t^{m}
$$

where $S_{n, 0}=0, S_{n, 1}=1$ and

$$
S_{n, m}=\frac{1}{m !} \sum_{1 \alpha_{2}+\cdots+(m-1) \alpha_{m}=m-1}(-1)^{\sum_{j=2}^{m} \alpha_{j}} \frac{\left(\sum_{j=2}^{m} j \alpha_{j}\right) !}{\prod_{j=2}^{m} \alpha_{j} !(j !)^{\alpha_{j}}} \prod_{j=2}^{m}\left(F^{(j)}(0)\right)^{\alpha_{j}}
$$

where $m=2,3, \ldots$

Proposition 1. Since the nontrivial Taylor series expansion coefficients of the function $F_{n}$ are $F_{n, 1}, F_{n, 2 n k+1}(k=1,2, \ldots)$, the nontrivial Taylor series expansion coefficients of the function $S_{n}$ are $S_{n, 1}, S_{n, 2 n k+1}(k=1,2, \ldots)$.

Therefore $S_{n}(t)=\sum_{k=0}^{\infty} S_{n, 2 n k+1} t^{2 n k+1}$ and we use in OZL-formula only those sums, which satisfy

$$
\sum_{j=1}^{k}(2 n j) \alpha_{2 n j+1}=2 n k \text { or } \sum_{j=1}^{k} j \beta_{j}=k, \text { where } \beta_{k}=\alpha_{2 n k+1} \quad(k=1,2, \ldots) .
$$

Then

$$
\begin{aligned}
S_{n, 2 n k+1}=\frac{1}{(2 n k+1) !} \sum_{1 \beta_{1}+\cdots+k \beta_{k}=k}(-1)^{\sum_{j=1}^{k} \beta_{j}} \\
\\
\times \frac{\left(\sum_{j=1}^{k}(2 n j+1) \beta_{j}\right) !}{\prod_{j=1}^{k} \beta_{j} !((2 n j+1) !)^{\beta_{j}}} \prod_{j=1}^{k}\left(F_{n}^{(2 n j+1)}(0)\right)^{\beta_{j}},
\end{aligned}
$$

or

$$
S_{n, 2 n k+1}=\frac{1}{2 n k+1} \sum_{1 \beta_{1}+\cdots+k \beta_{k}=k}(-1)^{\sum_{j=1}^{k} \beta_{j}}\left(V ; \beta_{1}, \ldots, \beta_{k}, 2 n k\right) \prod_{j=1}^{k} F_{n, 2 n j+1}^{\beta_{j}},
$$

where

$$
\sum_{j=1}^{k}(2 n j+1) \beta_{j}=2 n \sum_{j=1}^{k} j \beta_{j}+\sum_{j=1}^{k} \beta_{j}=2 n k+\sum_{j=1}^{k} \beta_{j}
$$

and 


$$
\left(V ; \beta_{1}, \ldots, \beta_{k}, 2 n k\right)=\frac{\left(2 n k+\sum_{j=1}^{k} \beta_{j}\right) !}{(2 n k) ! \prod_{j=1}^{k} \beta_{j} !}
$$

is a permutation with repetitions (cf. [1]) formula. If follows from (2.2) that

$$
\prod_{j=1}^{k} F_{n, 2 n j+1}^{\beta_{j}}=\prod_{j=1}^{k}\left(\frac{(2 j-1) ! !}{(2 j) ! !} \cdot \frac{1}{2 n j+1}\right)^{\beta_{j}} .
$$

One obtains that

$$
\begin{gathered}
\prod_{j=1}^{k}((2 j-1) ! !)^{\beta_{j}}=\prod_{j=1}^{k}\left(\frac{(2 j-1) ! !(2 j-2) ! !}{(2 j-2) ! !}\right)^{\beta_{j}}=\prod_{j=1}^{k}\left(\frac{(2 j-1) !}{(j-1) ! 2^{j-1}}\right)^{\beta_{i}} \\
=2^{-k+\sum_{j=1}^{k} \beta_{j}} \prod_{j=1}^{k}\left(\frac{(2 j-1) !}{(j-1) !}\right)^{\beta_{j}}, \\
\prod_{j=1}^{k}((2 j) ! !)^{\beta_{j}}=2^{k} \prod_{j=1}^{k}(j !)^{\beta_{j}}, \quad \prod_{j=1}^{k} F_{n, 2 n j+1}^{\beta_{j}}=2^{-2 k+\sum_{j=1}^{k} \beta_{j}} \frac{\prod_{j=1}^{k}\left(\begin{array}{c}
2 j-1 \\
j-1
\end{array}\right)^{\beta_{j}}}{\prod_{j=1}^{k}(2 n j+1)^{\beta_{j}}}
\end{gathered}
$$

where $\left(\begin{array}{c}2 k-1 \\ k-1\end{array}\right)(k=1,2, \ldots)$ are binomial coefficients (cf. [1]).

Notice that

$$
(-1)^{\sum_{j=1}^{k} \beta_{j}} 2^{-2 k+\sum_{j=1}^{k} \beta_{j}}=(-2)^{-2 k+\sum_{j=1}^{k} \beta_{j}} .
$$

The formula (2.1) follows from (2.3), (2.4) and (2.5).

Corollary 1 . The function $S_{1}(t)$ coincides with the elementary $\sin t$ with the well known nontrivial coefficients of the Taylor series expansion at $t=0$ :

$$
S_{1,2 k+1}=\frac{(-1)^{k}}{(2 k+1) !}
$$

Using (2.1) one obtains the set of identities:

$$
\sum_{1 \beta_{1}+\cdots+k \beta_{k}=k}(-2)^{-2 k+\sum_{i=1}^{k} \beta_{i}}\left(V ; \beta_{1}, \ldots, \beta_{k}, 2 k\right) \cdot \frac{\prod_{j=1}^{k}\left(\begin{array}{c}
2 j-1 \\
j-1
\end{array}\right)^{\beta_{j}}}{\prod_{j=1}^{k}(2 j+1)^{\beta_{j}}}=\frac{(-1)^{k}}{(2 k) !},
$$

where $k=1,2, \ldots$ 
Corollary 2. The function $S_{2}(t)$ coincides with the lemniscatic sine sl $t$ [3], which can be expressed through the Jacobian elliptic functions. Hence the nontrivial coefficients of the Taylor series expansion at $t=0$ for the lemniscatic sine are given by $S_{2,1}=1$ and

$$
\begin{aligned}
S_{2,4 k+1}=\frac{1}{4 k+1} \sum_{1 \beta_{1}+\cdots+k \beta_{k}=k}(-2)^{-2 k+\sum_{j=1}^{k} \beta_{j}} & \\
& \times\left(V ; \beta_{1}, \ldots, \beta_{k}, 4 k\right) \frac{\prod_{j=1}^{k}\left(\begin{array}{c}
2 j-1 \\
j-1
\end{array}\right)}{\prod_{j=1}^{k}(4 j+1)^{\beta_{j}}} \quad(k=1,2, \ldots) .
\end{aligned}
$$

\section{Verification}

\subsection{Function $\sin t$}

Consider $\sin t=1+\sum_{k=1}^{\infty} s_{2 k+1} t^{2 k+1}$. The values of $s_{2 k+1}(k=1,2, \ldots)$ obtained by computation using the formula (2.1) (for $n=1$ ) are

$$
s_{2 k+1}=\frac{(-1)^{k}}{(2 k+1) !}, \quad(k=1,2, \ldots) .
$$

\subsection{Function sl $t$}

Consider sl $t=\sum_{k=0}^{\infty} s_{4 k+1} t^{4 k+1}$. The first nontrivial coefficients of the Taylor series expansion for the function sl $t$ at $t=0$ given by the MAPLE program are:

$$
\begin{aligned}
& s_{1}=1, \quad s_{5}=-\frac{1}{10}, \quad s_{9}=\frac{1}{120}, \quad s_{13}=-\frac{11}{15600}, \\
& s_{17}=\frac{211}{3536000}, \quad s_{21}=-\frac{1607}{318240000} .
\end{aligned}
$$

As far as we know, the MAPLE program does not have explicit formulas for the Taylor coefficients of the Jacobian elliptic functions and calculates the series by repeatedly differentiating and evaluating at 0 (or whichever value we're expanding around). Of course it knows formulas for derivatives of the Jacobian elliptic functions.

The values of the coefficients $s_{4 k+1}(k=1,2, \ldots)$ above coincide with those obtained by computation using the formula (2.1) for $n=2$. Denote for brevity: 


$$
\begin{array}{ll}
A(\beta)=(-2)^{-2 k+\sum_{j=1}^{k} \beta_{j},} & B(\beta)=\left(V ; \beta_{1}, \ldots, \beta_{k}, 4 k\right), \\
C(\beta)=\prod_{j=1}^{k}\left(\begin{array}{c}
2 j-1 \\
j-1
\end{array}\right)^{\beta_{j}}, & D(\beta)=\prod_{j=1}^{k}(4 j+1)^{\beta_{j}} .
\end{array}
$$

3.2.1. $k=1$

$$
\begin{array}{ccccc}
\multicolumn{5}{c}{1 \cdot \beta_{1}=1 .} \\
\hline \beta_{1} & A(\beta) & B(\beta) & C(\beta) & D(\beta) \\
\hline 1 & -\frac{1}{2} & 5 & 1 & 5 \\
\hline s_{5} & =s_{4 \cdot 1+1}=\frac{1}{5} \cdot\left(-\frac{1}{2}\right) \cdot 5 \cdot \frac{1}{5}=-\frac{1}{10} .
\end{array}
$$

\begin{tabular}{ccccc}
\hline$\beta_{1}$ & $A(\beta)$ & $B(\beta)$ & $C(\beta)$ & $D(\beta)$ \\
\hline 1 & $-\frac{1}{2}$ & 5 & 1 & 5 \\
\hline
\end{tabular}

3.2.2. $k=2$

$$
\begin{aligned}
& 1 \cdot \beta_{1}+2 \cdot \beta_{2}=2 . \\
& \begin{array}{cccccc}
\hline \beta_{1} & \beta_{2} & A(\beta) & B(\beta) & C(\beta) & D(\beta) \\
\hline 2 & 0 & \frac{1}{4} & 45 & 1 & 25
\end{array} \\
& \begin{array}{llllll}
0 & 1 & -\frac{1}{8} & 9 & 3 & 9 \\
\hline
\end{array} \\
& s_{9}=s_{4 \cdot 2+1}=\frac{1}{9}\left[\frac{1}{4} \cdot 45 \cdot \frac{1}{25}+\left(-\frac{1}{8}\right) \cdot 9 \cdot \frac{3}{9}\right]=\frac{1}{120} .
\end{aligned}
$$

3.2.3. $k=3$

$$
\begin{aligned}
& 1 \cdot \beta_{1}+2 \cdot \beta_{2}+3 \cdot \beta_{3}=3 . \\
& \begin{array}{ccccccc}
\hline \beta_{1} & \beta_{2} & \beta_{3} & A(\beta) & B(\beta) & C(\beta) & D(\beta) \\
\hline 3 & 0 & 0 & -\frac{1}{8} & 455 & 1 & 125 \\
0 & 0 & 1 & -\frac{1}{32} & 13 & 10 & 13 \\
1 & 1 & 0 & \frac{1}{16} & 182 & 3 & 45 \\
\hline
\end{array} \\
& s_{13}=s_{4 \cdot 3+1}=\frac{1}{13}\left[\left(-\frac{1}{8}\right) \cdot 455 \cdot \frac{1}{125}+\left(-\frac{1}{32}\right) \cdot 13 \cdot \frac{10}{13}\right. \\
& \left.+\frac{1}{16} \cdot 182 \cdot \frac{3}{45}\right]=-\frac{11}{15600} .
\end{aligned}
$$


3.2.4. $k=4$

$$
\begin{array}{ccccccccc}
\multicolumn{8}{c}{1 \cdot \beta_{1}+2 \cdot \beta_{2}+3 \cdot \beta_{3}+4 \cdot \beta_{4}=4 .} \\
\cline { 2 - 8 } & \beta_{1} & \beta_{2} & \beta_{3} & \beta_{4} & A(\beta) & B(\beta) & C(\beta) & D(\beta) \\
\hline 4 & 0 & 0 & 0 & \frac{1}{16} & 4845 & 1 & 625 \\
0 & 0 & 0 & 1 & -\frac{1}{128} & 17 & 35 & 17 \\
2 & 1 & 0 & 0 & -\frac{1}{32} & 2907 & 3 & 225 \\
1 & 0 & 1 & 0 & \frac{1}{64} & 306 & 10 & 65 \\
0 & 2 & 0 & 0 & \frac{1}{64} & 153 & 9 & 81 \\
\hline s_{17}=s_{4 \cdot 4+1}= & \frac{1}{17}\left[\frac{1}{16} \cdot 4845 \cdot \frac{1}{625}-\frac{1}{128} \cdot 17 \cdot \frac{35}{17}-\frac{1}{32} \cdot 2907 \cdot \frac{3}{225}\right. \\
\end{array} .
$$

3.2.5. $k=5$

\begin{tabular}{rccccrrrrr}
\multicolumn{10}{c}{$1 \cdot \beta_{1}+2 \cdot \beta_{2}+3 \cdot \beta_{3}+4 \cdot \beta_{4}+5 \cdot \beta_{5}=5}$. \\
\hline$\beta_{1}$ & $\beta_{2}$ & $\beta_{3}$ & $\beta_{4}$ & $\beta_{5}$ & $A(\beta)$ & $B(\beta)$ & $C(\beta)$ & $D(\beta)$ \\
\hline 5 & 0 & 0 & 0 & 0 & $-1 / 32$ & 53130 & 1 & 3125 \\
3 & 1 & 0 & 0 & 0 & $1 / 64$ & 42504 & 3 & 1125 \\
2 & 0 & 1 & 0 & 0 & $-1 / 128$ & 5313 & 10 & 325 \\
1 & 0 & 0 & 1 & 0 & $1 / 256$ & 462 & 35 & 85 \\
0 & 1 & 1 & 0 & 0 & $1 / 256$ & 462 & 30 & 117 \\
0 & 0 & 0 & 0 & 1 & $-1 / 512$ & 21 & 126 & 21 \\
1 & 2 & 0 & 0 & 0 & $-1 / 128$ & 5313 & 9 & 405 \\
\hline
\end{tabular}

$$
\begin{aligned}
s_{21}=s_{4 \cdot 5+1}= & \frac{1}{21}\left[-\frac{1}{32} \cdot 53130 \cdot \frac{1}{3125}+\frac{1}{64} \cdot 42504 \cdot \frac{3}{1125}-\frac{1}{128} \cdot 5313 \cdot \frac{10}{325}\right. \\
& +\frac{1}{256} \cdot 462 \cdot \frac{35}{85}+\frac{1}{256} \cdot 462 \cdot \frac{30}{117}-\frac{1}{512} \cdot 21 \cdot \frac{126}{21} \\
& \left.+\left(-\frac{1}{128}\right) \cdot 5313 \cdot \frac{9}{405}\right]=-\frac{1607}{318240000} .
\end{aligned}
$$




\section{Conclusions}

In this article we study the functions $S_{n}(t)$ defined as solutions of the following Cauchy problem

$$
\left\{\begin{array}{l}
x^{\prime \prime}=-n x^{2 n-1}, \\
x(0)=0, \quad x^{\prime}(0)=1
\end{array}\right.
$$

and their Taylor series expansions around the point $t=0$. For $n=1$ one obtains the $\sin t$ function, the case $n=2$ refers to the lemniscatic sine function sl $t$. Integrating the differential equation and making use of the initial conditions one gets the integral representation

$$
t=\int_{0}^{x} \frac{d u}{\sqrt{1-u^{2 n}}}, \quad x=S_{n}(t) .
$$

The function under the integral can be expanded in a Taylor series with respect to $u$ and then integrated. Thus the Taylor series expansion for the function $F_{n}(x)$, which is defined as an inverse to $S_{n}(t)$, can be obtained. We were able to compute the coefficients of the Taylor series expansion around zero of the functions $S_{n}(t)(n=1,2, \ldots)$ making use of the Ostrowski-Zabreiko-Lysenko formula [12] for the higher derivatives of an inverse function. This formula is stated as the main result of the article in Theorem 1. Manual computation of the coefficients up to the 21-st term in the Taylor series expansion of the lemniscatic sine function sl $t$ was carried out.

\section{References}

[1] M. Abramowitz and I. Stegun(Eds.). Handbook of mathematical functions. Nat. Bureau of Standards, 1964. (Russian edition: Nauka, Moscow, 1979)

[2] D. Dumont. A combinatorial interpretation for the Schett recurrence on the Jacobian elliptic functions. Math. Comp., 33, 1293 - 1297, 1979.

[3] A. Gritsans and F. Sadyrbaev. Lemniscatic functions in the theory of the Emden - Fowler differential equation. Mathematics. Differential equations (Univ. of Latvia, Institute of Math. and Comp. Sci.), 3, 2003.

[4] Hyo Chul Myung. Note on the Jacobi elliptic functions. J. Algebra, 200, 134 $140,1998$.

[5] A.M. Ostrowski. Solutions of equations and systems of equations. Acad. Press: New York and London, 1960. (Russian edition, Moscow, 1963)

[6] A. Schett. Properties of the Taylor series expansion coefficients of the Jacobian elliptic functions. Math. Comp., 30, $143-147,1976$.

[7] A. Schett. Recurrence formula of the Taylor series expansion coefficients of the Jacobian elliptic functions. Math. Comp., 31, 1003 - 1005, 1977.

[8] Corrigendum to A. Schett. Properties of the Taylor series expansion coefficients of the Jacobian elliptic functions, Math. Comp. 30 (1976), 143 - 147. Math. Comp., 31, 330, 1977.

[9] E.T. Whittaker and G. N. Watson. A Course of Modern Analysis, Part II. Cambridge Univ. Press, 1927. 
[10] S. Wrigge. Calculation of the Taylor series expansion coefficients of the Jacobian elliptic function $\operatorname{sn}(x, k)$. Math. Comp., 36, $555-564,1981$.

[11] S. Wrigge. A note on the Taylor series expansion coefficients of the Jacobian elliptic function $\operatorname{sn}(x, k)$. Math. Comp., 37, 495-497, 1981.

[12] P.P. Zabreiko and Y. V. Lysenko. Exact formulas for higher order derivatives for inverse functions in Banach spaces. Doklady of the national academy of sciences of Belarus, 45, $27-30,2001$.

Emdeno-Flauerio tipo lygčių sprendinių dèstinių Teiloro eilutėmis koeficientai

A. Gritsans, F. Sadyrbaev

Pateikiamos vadinamos Emdeno-Faulerio lygties $x^{\prime \prime}=-n x^{2 n-1}$ pradinio uždavinio $x(0)=0, x^{\prime}(0)=1,(n=1,2, \ldots)$ sprendiniu $S_{n}(t)$ Teiloro eilute koeficientu išreikštinès formulès. Jos yra nerekursyvinè. 